\title{
Intrinsic limitations in the energy resolution of drift-field based radiation detectors: a Monte Carlo simulation study of Xe-filled counters
}

\author{
L.M.N. Távora ${ }^{\mathrm{a}, \mathrm{b}, *}$, C.A.N. Conde ${ }^{\mathrm{b}}$, F.P. Santos ${ }^{\mathrm{b}}$, T.H.V.T. Dias ${ }^{\mathrm{b}}$, \\ P.J.B.M. Rachinhas \\ ${ }^{a}$ ESTG, Instituto Politécnico de Leiria, Morro do Lena-Alto do Vieiro, 2411-901 Leiria, Portugal \\ ${ }^{\mathrm{b}}$ Centro de Instrumentação, Departamento de Física da Universidade de Coimbra, Coimbra, Portugal
}

Many radiation detectors rely on the use of an electric field to drift the charged particles resulting from the interaction of the incoming radiation with the medium (Knoll, 1999). When migrating, and between collisions with the medium's atoms, they exchange energy with the external electric field, increasing/reducing their kinetic energy, thus increasing/reducing the total energy dissipated in the medium. This effect may introduce fluctuations in the energy and number of secondary particles being produced (Rachinhas et al., 1999).

The response of Xe-based radiation detectors has been studied using Monte Carlo techniques in order to assess the total energy deposited in the detector, as this quantity should give a fairly good indication in what respects the degradation of the detector's energy resolution. The detector set-up is an $L=5 \mathrm{~cm}$ long absorption region filled with Xe at $1 \mathrm{~atm}$. Photoelectron trajectories in the medium, under the external electric field, were simulated with the PENELOPE code (Salvat et al., 2001) until their energy fell below $150 \mathrm{eV}$.

The total energy deposited by photons with energies of 60,100 and $200 \mathrm{keV}$ in this detector has been studied. Reduced electric fields $(E / p)$, ranging from 0.1 to $0.8 \mathrm{~V} \mathrm{~cm}^{-1}$ Torr $^{-1}$ (fields commonly used to drift charged particles in gas detectors) have been considered, for each photon energy. The histograms describing the total energy deposition are shown in Fig. 1(a)-(c). At each photon energy $E$, the importance of the drift

*Corresponding author. ESTG, Instituto Politécnico de Leiria, Morro do Lena-Alto do Vieiro, 2411-901 Leiria, Portugal.

E-mail address: 1mtavora@estg.ipleiria.pt (L.M.N. Távora). electric field effect in the deterioration of the energy resolution can be assessed by comparing the histograms with the Gaussian curve labelled "Intrinsic", whose width is associated only to intrinsic fluctuations in the production of electrons in the $\mathrm{Xe}$ medium, i.e. $\mathrm{FWHM}=2.35(F w E)^{1 / 2}$, where $w=E / \bar{n}$ is the mean energy required to produce a subionization electron in the gas, $F$, the so-called Fano factor, is the relative variance $F=\sigma^{2} / \bar{n}$, and $\bar{n}$ is the mean number of electrons produced per X-ray photon of energy $E$ (Knoll, 1989).

The data presented make it clear that drift electric fields do affect the total energy that is deposited in the detector. Also, the spread of the data in the curves is seen to increase with the magnitude of the applied electric field and the energy of the incoming photons. This can be understood on the following grounds: under higher electric fields, the increased potential variations experienced by electrons result in more significant energy transfers to/from the field. Additionally, in regimes where photoelectric absorption dominates, higher photon energies produce faster photoelectrons and, since the electron scattering cross sections generally decrease with energy (Perkins et al., 1991), faster electrons will travel longer paths between collisions, thus exchanging more energy with the field. The fact that electrons with energies below $150 \mathrm{eV}$ are not transported and their contributions are neglected can, at the most, result in an underestimation of the results. However, the simulations have shown that the observed spreads are mainly related to the longer paths followed by the higher energy electrons, so this $150 \mathrm{eV}$ energy cut should not greatly affect the results obtained. At higher energies, the angular distribution of emitted photoelectrons will 

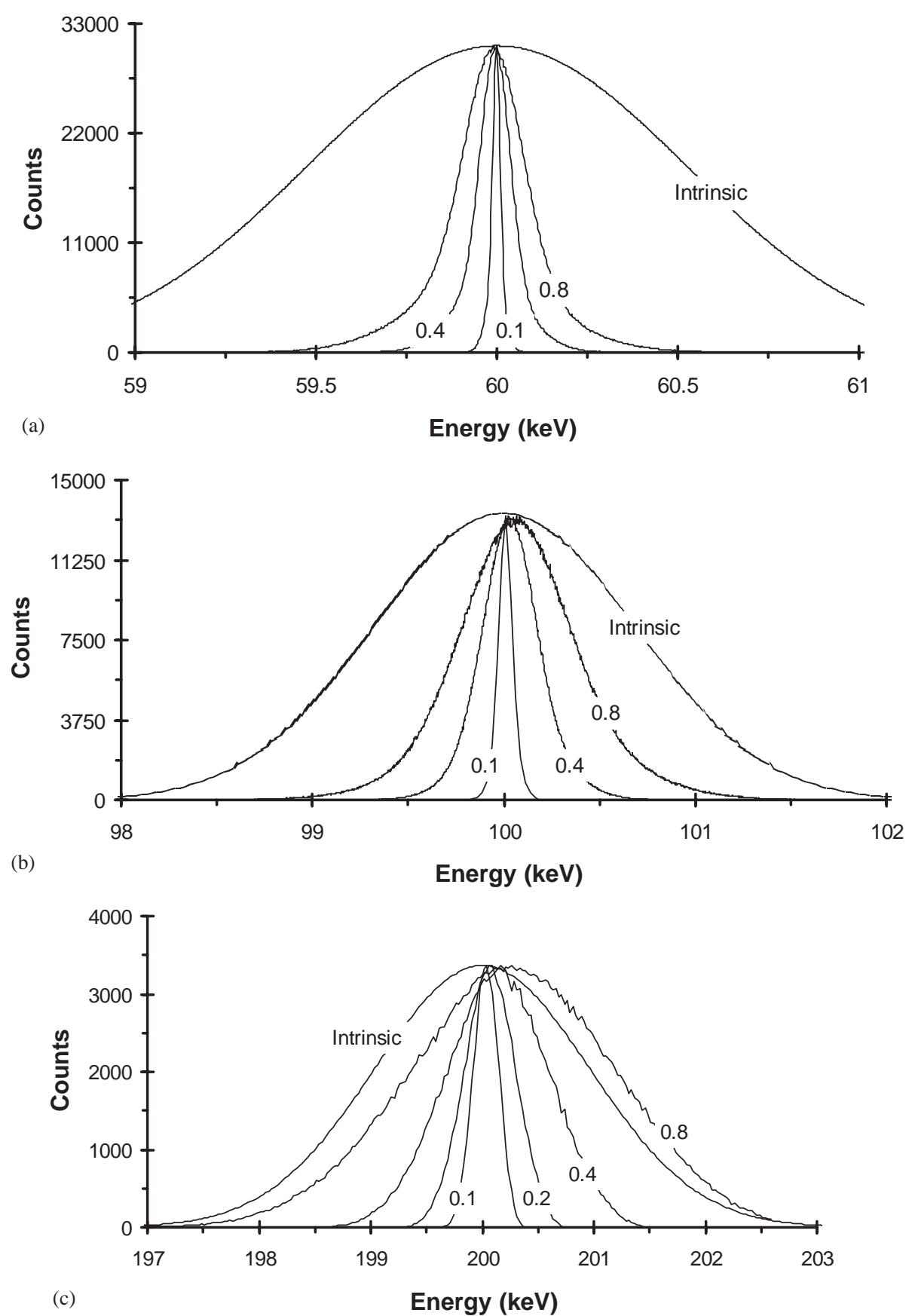

Fig. 1. Energy deposited in a Xe-based radiation detector by photons of (a) $60 \mathrm{keV}$, (b) $100 \mathrm{keV}$ and (c) $200 \mathrm{keV}$ : histograms showing the spread in the full-energy peak for the indicated drift electric fields (units are $\mathrm{V} \mathrm{cm}^{-} 1 \mathrm{Torr}^{-} 1$ ).

peak close to zero degrees and electron trajectories will start in directions almost opposite to the field. In these circumstances, electrons will, on average, gain (rather than lose) energy from the field and so, at increasing photon energies and electric fields, a progressive shift of the energy distributions mean values towards energies higher than $E$ is clearly observed.
Simulation studies carried out have shown that, to some extent, the spread observed in curves presented is mainly due to energy transfer mechanisms undertaken by the primary photoelectron. Again, being the most energetic electron in the medium and giving away only a small fraction of its energy in each collision, it will travel longer distances, experiencing higher energy variations. 
In conclusion, for energies above $\sim 100 \mathrm{keV}$ the effect of the field, in terms of fluctuations observed in the total energy deposited in the detector, may indeed result in an overall degradation of the spectroscopic capabilities of the system.

The kind of behaviour observed in the results presented here should, in principle, occur in all gas and semiconductor detectors that make use of drift electric fields. However higher atomic densities result in much smaller paths between collisions, so that energy exchanges with the field are much lower, making this effect negligible in terms of the solid state detector's performance.

\section{Acknowledgements}

The authors acknowledge financial support from FEDER, Fundção para a Ciência e Tecnologia (through
Project POCTI/FNU/49561/2002) and Fundação Calouste Gulbenkian.

\section{References}

Knoll, G.F., 1999. Radiation Detection and Measurement 3rd Edition. Wiley, New York.

Perkins, S.T., Cullen, D.E., Seltzer, S.M., 1991. Tables and graphs of electron-interaction cross sections from $10 \mathrm{eV}$ to $100 \mathrm{GeV}$ derived from the LLNL evaluated electron data library (EEDL), $Z=1-100$. Lawrence Livermore National Laboratory Report UCRL-50400 Vol. 31.

Rachinhas, P.J.B.M., et al., 1999. Absorption of electrons in Xenon for Energies up to $200 \mathrm{kev}$ : a Monte Carlo simulation study. IEEEE Trans. Nucl. Sci. 46, 1898-1900.

Salvat, F., et al, 2001. PENELOPE, a code system for Monte Carlo simulation of electron and photon transport, OECD/ NEA 5-7 November 2001, NEA/NSC/DOC, 2001, pp. 19 . 